\title{
CLASSIFICAÇÃO DE PACIENTES INTERNADOS EM UMA UNIDADE TRAUMATOLÓGICA
}

\author{
Monica de MORAES ${ }^{\mathrm{a}}$, Graciele Fernanda da Costa LINCH ${ }^{\mathrm{b}}$, \\ Emiliane Nogueira SOUZA ${ }^{\mathrm{C}}$
}

\section{RESUMO}

Estudo com o objetivo de classificar pacientes de acordo com o grau de dependência da assistência de enfermagem, dimensionar a equipe de enfermagem e correlacionar os instrumentos utilizados. Foram utilizados os instrumentos de Perroca e Fugulin para classificar os pacientes internados durante 30 dias. O dimensionamento da equipe de enfermagem foi realizado conforme recomendação do Conselho Federal de Enfermagem. Incluiu-se 157 pacientes, sendo 42,0\% classificados com o grau de dependência de cuidados intermediários e, em 50,3\% dos pacientes, o grau de dependência foi classificado como "cuidados mínimos". Em relação ao dimensionamento da equipe de enfermagem, o quadro de funcionários existente no setor pesquisado era de 20 funcionários, e o necessário seria de 32 funcionários, divididos nos quatro turnos. Os instrumentos apresentaram uma forte correlação $\left(r_{p}=0,88\right)$. Acredita-se que a classificação de pacientes com vistas ao dimensionamento da equipe de enfermagem pode oferecer mais qualidade ao cuidado.

Descritores: Enfermagem. Cuidados de enfermagem. Classificação. Gerenciamento do tempo. Downsizing organizacional.

\section{RESUMEN}

Estudio cuyo objetivo es clasificar pacientes según el grado de dependencia de la asistencia de enfermeira, el tamaño del personal de enfermería y correlacionar los instrumentos. Se utilizaron los instrumentos de Perroca y Fugulin para clasificar los pacientes internados por un periodo de 30 días, y, para realizar el dimensionamiento del equipo, fue utilizada la fórmula recomendada por la Resolución 239/O4 del Consejo Federal de Enfermería (COFEN). Se incluyeron 157 pacientes, el 42,0\% fue clasificada con el grado de dependencia de cuidados intermediarios y, en un 50,3\% de los pacientes, el grado de dependencia fue clasificado como cuidados mínimos. Con relación al dimensionamiento del equipo de enfermería, el número de empleados existente en el sector investigado era de 20 empleados y lo necesario serían 32 en total, divididos en cuatro turnos. Dichos pacientes fueron evaluados por dos instrumentos que presentaron fuerte correlación $\left(r_{p}=0,88\right)$. Se cree que la clasificación de pacientes puede relacionarse con un buen equipo de enfermería, ya que un número mayor ofrece más calidad al cuidado.

Descriptores: Enfermería. Atención de enfermería. Clasificación. Administración del tiempo. Reducción de personal. Título: Clasificación de pacientes internados en una unidad traumatológica.

\section{ABSTRACT}

Study with the aim to classify patients in accordance with the degree of dependence on nursing care, estimate the size of the nursing staff and correlate instruments. Perroca and Fugulin's instruments were used to classify patients admitted for a period of 30 days and to estimate the size of the nursing staff, the formula recommended by the Federal Nursing Board COFEN No. 293/O was used. Included were 157 patients, $42.0 \%$ were classified as dependent on intermediate care, and for 50.3\% of patients, the degree of dependence was classified as minimum care. Regarding the size of the nursing staff, the existing staff in the surveyed unit consisted of 20 employees, while the necessary size should be 32 employees, divided in the four shifts. The instruments showed a strong correlation $\left(r_{p}=0.88\right)$. It is believed that the classification of patients in order to estimate the size of the nursing team can provide more quality to care.

Descriptors: Nursing. Nursing care. Classification. Time management. Personnel downsizing.

Title: Classification of patients in a traumatology unit.

\footnotetext{
a Enfermeira graduada pela Faculdade Fátima, Caxias do Sul - RS, Brasil.

b Mestre em Enfermagem, Bolsista CAPES, Doutoranda, Escola de Enfermagem, UFRGS, Porto Alegre, RS, Brasil.

c Doutoranda em Ciência da Saúde: cardiologia e ciências cardiovasculares UFRGS. Docente do Departamento de Enfermagem da Universidade Federal de Ciências da Saúde de Porto Alegre (UFCSPA).
} 


\section{INTRODUÇÃO}

A necessidade de classificação do grau de dependência dos pacientes vem se tornando uma prioridade, com estes dados é possível prever aspectos relacionados ao processo assistencial, bem como assegurar o adequado dimensionamento de profissionais de enfermagem necessários para prestar os cuidados aos pacientes ${ }^{(1)}$. A ideia de classificar paciente não é recente; Florence Nightingale se utilizou desta prática, buscando localizar, na enfermaria, os pacientes cujo nível de cuidado demandava maior atenção ${ }^{(2)}$.

No contexto hospitalar, os pacientes ficam dependentes de cuidados, o que torna os membros da equipe de enfermagem elementos fundamentais na assistência à saúde ${ }^{(1)}$. Para tal, torna-se necessária uma assistência de enfermagem individualizada, integral e sistematizada, a partir de um exame clínico rigoroso, e posterior classificação do grau de dependência do paciente para os cuidados. Um sistema de classificação de pacientes objetiva equalizar a relação demanda (paciente) e oferta de cuidado (trabalhadores de enfermagem), de forma que o cuidado seja prestado conforme a necessidade do paciente, sem que isso traga sobrecarga ao trabalhador.

Pesquisadoras construíram um instrumento para classificação de pacientes baseado na Teoria das Necessidades Humanas Básicas, preconizadas por Horta em 1979, considerando 13 indicadores $\operatorname{críticos}^{(3)}$. Esses são classificados dentro de cinco variáveis, e o somatório final categoriza os cuidados em: mínimos, intermediários, semi-intensivos e intensivos. As mesmas autoras realizaram outro estudo em que participaram 15 enfermeiros com experiência profissional, para analisarem a adequação dos indicadores para validação da escala de classificação do grau de dependência dos pacientes. Diante dos resultados, esse estudo permitiu concluir que o instrumento apresenta condições de ser aplicado na prática gerencial do enfermeiro, sendo o mesmo um norteador das necessidades de cuidado do paciente, bem como da carga de trabalho da equipe de enfermagem ${ }^{(3)}$.

Da mesma forma, outro instrumento foi elaborado para a classificação que estabelece nove áreas de cuidados ${ }^{(4)}$. A partir dessa avaliação, os pacientes são classificados em uma das seguintes categorias: cuidados intensivos, cuidados semi-intensivos, cuidados de alta dependência, cuidados intermediários e cuidados mínimos.
Ao considerar os diferentes graus de complexidade assistencial em unidades de internação, a enfermeira tem subsídios para buscar a adequação dos recursos humanos e materiais de forma crítica, reflexiva e consonante com a realidade, o que pode gerar melhorias na qualidade da assistência de enfermagem ${ }^{(4)}$. Tal instrumento é referendado pela Resolução do Conselho Federal de Enfermagem (COFEN) no. 189/96. Para homogeneizar sua aplicação, foram atribuídos pesos a cada nível de dependência em relação às áreas de cuidado. A soma dos valores obtidos em cada área e a definição de cada categoria de cuidados determina a complexidade assistencial do paciente ${ }^{(4)}$.

Para os pacientes traumatológicos, no período pré e pós-operatório, que sofrem o impacto da redução ou mesmo perda das funções vitais e se tornam dependentes de cuidados de enfermagem, é pertinente adotar um sistema para classificar seu grau de dependência. Isso visa contribuir para que estes pacientes recebam o cuidado adequado e com a qualidade que efetivamente necessitam, a partir da alocação adequada dos membros da equipe de enfermagem e para que os trabalhadores não tenham sobrecarga de trabalho.

Dessa forma, são necessários estudos específicos sobre o grau de dependência do paciente com trauma, assim optou-se por aplicar os instrumentos mencionados para a classificação dos pacientes em uma unidade traumatológica, devido à importância de se conhecer as características dos pacientes com relação à necessidade de cuidados de enfermagem ${ }^{(3,4)}$, para evitar a sobrecarga de trabalho, e conseqüente, queda da qualidade assistencial. Soma-se a isso o interesse em oferecer subsídios para uma melhor adequação dos recursos humanos para o cuidado desses pacientes.

O prognóstico desses pacientes está diretamente relacionado à qualidade da assistência e à agilidade com que a mesma é prestada. Geram-se, assim, indicadores de bons resultados a médio e longo prazo, a partir do trabalho da enfermagem. Na literatura, os sistemas de classificação de pacientes têm sido amplamente utilizados, mas não há divulgação de estudos com pacientes traumatológicos.

Nesse contexto, este estudo foi delineado para classificar pacientes de acordo com o grau de dependência da assistência de enfermagem, dimensionar a equipe de enfermagem e correlacionar os instrumentos utilizados. 


\section{MATERIAIS E MÉTODOS}

Trata-se de um estudo com delineamento transversal, realizado em um hospital filantrópico de grande porte, referência no atendimento ao paciente com trauma na região nordeste do Rio Grande do Sul, Brasil.

A população em estudo foi composta por pacientes que sofreram algum tipo de trauma, internados em unidade traumatológica, durante o período de 30 dias (março de 2010). É importante salientar que, diariamente, a totalidade de pacientes internados foi avaliada, independente de um mesmo paciente já ter sido observado nos dias anteriores. Para tanto, a amostra deste estudo foi definida de acordo com o número de pacientes internados na unidade no período da coleta de dados. Foram elegíveis para o estudo sujeitos internados no setor, mediante assinatura do Termo de Consentimento Livre e Esclarecido. Dessa maneira, foram incluídos 157 pacientes nesse estudo.

Para classificação dos pacientes quanto à dependência dos cuidados de enfermagem, foram utilizados dois instrumentos: o sistema de classificação de Perroca ${ }^{(2)}$ e o instrumento de classificação de pacientes de Fugulin ${ }^{(4)}$.

O Sistema Classificação de Perroca ${ }^{(2)}$ é composto por 13 indicadores críticos de cuidados (necessidades de cuidado de enfermagem do paciente): estado mental e nível de consciência, oxigenação, sinais vitais, nutrição e hidratação, motilidade, locomoção, cuidado corporal, eliminações, terapêutica, educação à saúde, comportamento, comunicação e integridade cutânea mucosa. Cada um dos indicadores possui uma graduação que varia de um a cinco, apontando a intensidade crescente de complexidade do cuidado, de forma que o valor 1 corresponde ao menor nível de complexidade assistencial, e o valor 5, ao nível máximo de complexidade assistencial. Para análise, o valor obtido individualmente, em cada um dos indicadores, é somado, e o valor total é obtido comparado com os intervalos de pontuações propostos, conduzindo, dessa forma, a uma classe ou categoria de cuidado a que esse paciente pertence: cuidados mínimos (13 a 26 pontos), cuidados intermediários (27 a 39 pontos), cuidados semi-intensivos (40 a 52 pontos) e cuidados intensivos (53 a 65 pontos)

O Instrumento de Classificação de Fugulin ${ }^{(4)}$ estabelece nove áreas de cuidados, a saber: estado mental, oxigenação, sinais vitais, motilidade, deambulação, alimentação, cuidado corporal, eliminação e terapêutica, de acordo com a complexidade assistencial dos pacientes internados. A partir dessa avaliação, os pacientes são classificados em uma das categorias: cuidados intensivos (acima de 31 pontos), cuidados semi-intensivos (27 a 31 pontos), cuidados de alta dependência (21 a 26 pontos), cuidados intermediários (15 a 20 pontos) e cuidados mínimos (9 a 14 pontos).

Além desses instrumentos, elaborou-se um questionário com as variáveis sociodemográficas e clínicas. A coleta de dados foi realizada diariamente. Todos os pacientes internados foram avaliados por meio de observação e, quando necessário, pelo diálogo com paciente e familiar.

Os resultados foram analisados por meio de estatística descritiva e analítica. As variáveis categóricas foram expressas com valores absolutos (n) e relativos (\%), e as variáveis contínuas descritas como média \pm desvio padrão ou mediana e percentis 25 e 75, conforme seguissem ou não a distribuição normal. As análises foram realizadas utilizando o pacote estatístico Statistical Package for Social Sciences (SPSS) 17.0. A correlação entre as classificações foi realizada com a utilização do coeficiente de correlação de Spearman $\left(r_{\mathrm{p}}\right)$.

Para o cálculo do dimensionamento da equipe de enfermagem, foi utilizado a Resolução Conselho Federal de Enfermagem (COFEN) nº. 293/2004, que menciona a metodologia de cálculo de pessoal de enfermagem ${ }^{(5)}$ : quantidade de pessoal $(\mathrm{QP})$ é o número de profissionais de enfermagem necessário na unidade de internação, com base no sistema de classificação de pacientes e na taxa de ocupação; total de horas de enfermagem (THE) é o somatório das horas necessárias para assistir os clientes com demanda de cuidados mínimos, intermediários, semi-intensivos e intensivos; constante de marinho (KM) significa o coeficiente deduzido em função de dias da semana (DS), da jornada semanal de trabalho (JST), que assume os valores de 40h nas unidades assistenciais. E o índice de segurança técnica (IST) é composto de duas parcelas fundamentais, a taxa de ausências por benefícios (planejada, isto é, para cobertura de férias, licenças-gestante, etc.) e a taxa de absenteísmo (não planejada, ou seja, para cobertura de ausências / faltas, por diversos motivos).

Utilizando-se o coeficiente IST igual a 1,15 (15\%), e substituindo JST pelos seus valores assumidos de 40 horas, a KM terá o valor respectivo de: $\mathrm{KM}_{(40)}=0,2012$. 
Para a variável dimensionamento de funcionários, foram utilizadas as horas médias de assistência de enfermagem preconizadas pela resolução COFEN 293/04 ${ }^{(5)}$, acrescentando, para os pacientes que requerem cuidados de alta dependência (CAD), conforme o instrumento de Perroca ${ }^{(2)}$, uma estimativa de seis horas, no cálculo do THE, uma vez que esta resolução não contempla esse tipo de cuidado. Para efeito de cálculo, devem ser consideradas como horas de Enfermagem, por leito, nas 24 horas: 3,8 horas de Enfermagem, por cliente, na assistência mínima ou em condições de autocuidado; 5,6 horas de Enfermagem, por cliente, na assistência intermediária; 9,4 horas de Enfermagem, por cliente, na assistência semi-intensiva; 17,9 horas de Enfermagem, por cliente, na assistência intensiva. Sendo os pacientes distribuídos da seguinte forma: pacientes com cuidados mínimos (PCM); pacientes com cuidados intermediários (PCI); pacientes com cuidados semi-intensivos (PCSI); pacientes com cuidados intensivos (PCIT). A partir disso, tem-se a fórmula do THE:

$$
\begin{gathered}
\text { THE }=[(\text { PCM } \times 3,8)+(\text { PCI x 5,6) } \\
(\text { PCSI } \times 9,4)+(\text { PCIT } \times 17,9)]
\end{gathered}
$$

E, finalmente, substituindo KM e THE na equação abaixo, serão obtidos as correspondentes quantidades do pessoal de enfermagem.

$$
\mathrm{QP}=\mathrm{KM} \times \text { total de horas de enfermagem }
$$$$
\text { (THE) }
$$

A distribuição percentual do total de profissionais de Enfermagem deve contemplar as proporções, de acordo com o sistema de classificação de pacientes: cuidados mínimos e intermediários: de 33 a 37\% são enfermeiros e, os demais, técnicos de enfermagem; para assistência semi-intensiva: de 42 a $46 \%$ são enfermeiros e os demais, técnicos e auxiliares de enfermagem; para assistência intensiva: de 52 a $56 \%$ são enfermeiros e os demais, técnicos de enfermagem ${ }^{(5)}$.

O projeto de pesquisa foi enviado para o Comitê de Ética e Pesquisa da instituição, sendo aprovado sob o número 089/2010.

\section{RESULTADOS}

Foram incluídos 157 pacientes, e avaliados quanto ao grau de dependência dos cuidados de enfermagem em 30 dias. As características sociodemográficas e clínicas estão demonstradas na Tabela 1.
Em relação ao escore de classificação do instrumento de Fugulin, o escore médio dos 157 pacientes avaliados foi de $18,74 \pm 4,36$, cuja complexidade assistencial foi classificada como cuidados intermediários.

Referente ao escore de classificação de Perroca, os pacientes avaliados apresentaram o escore médio de $27,04 \pm 6,56$, classificados como cuidados intermediários.

Na Figura 1, de acordo com o sistema de classificação de Perroca, é possível observar a distribuição dos pacientes conforme a complexidade, destaca-se que nenhum paciente foi classificado em cuidados intensivos.

Na Figura 2, pode-se observar a distribuição dos pacientes segundo o instrumento de classificação de Fugulin.

Ao ser avaliada a correlação entre os escores de Fugulin e Perroca identificou-se forte correlação $\left(\mathrm{r}_{\mathrm{p}}=0,88\right)$, ou seja, à medida que o paciente teve seu escore de classificação de Fugulin aumentado, o escore de classificação de Perroca também aumentou, evidenciando uma correlação direta.

Tabela 1 - Características sociodemográficas da amostra. Caxias do Sul, RS, 2010.

\begin{tabular}{ll}
\hline Variável & $\mathbf{n}(\%)$ \\
\hline Sexo masculino & $114(72,6)$ \\
Idade $(\mathrm{m} \pm \mathrm{dp})$ & $45,29 \pm 18,49$ \\
Tempo de permanência* & $4(3-7,5)$ \\
Motivo da internação: & \\
$\quad$ Acidentes automobilísticos & $58(36,9)$ \\
$\quad$ Cirurgias eletivas & $57(36,3)$ \\
$\quad$ Queda ao solo & $42(26,8)$ \\
Diagnóstico principal: & \\
$\quad$ Fraturas & $72(45,9)$ \\
$\quad$ Pós-operatório & $41(26,1)$ \\
$\quad$ Pré-operatório & $14(8,9)$ \\
$\quad$ Outros & $30(18,9)$ \\
Comorbidades: & \\
$\quad$ Hipertensão arterial sistêmica & $26(16,6)$ \\
$\quad$ Diabetes melitus & $3(1,9)$ \\
$\quad$ Acidente vascular cerebral & $2(1,3)$ \\
\hline
\end{tabular}

* Dado apresentado com mediana e percentil 25-75. 


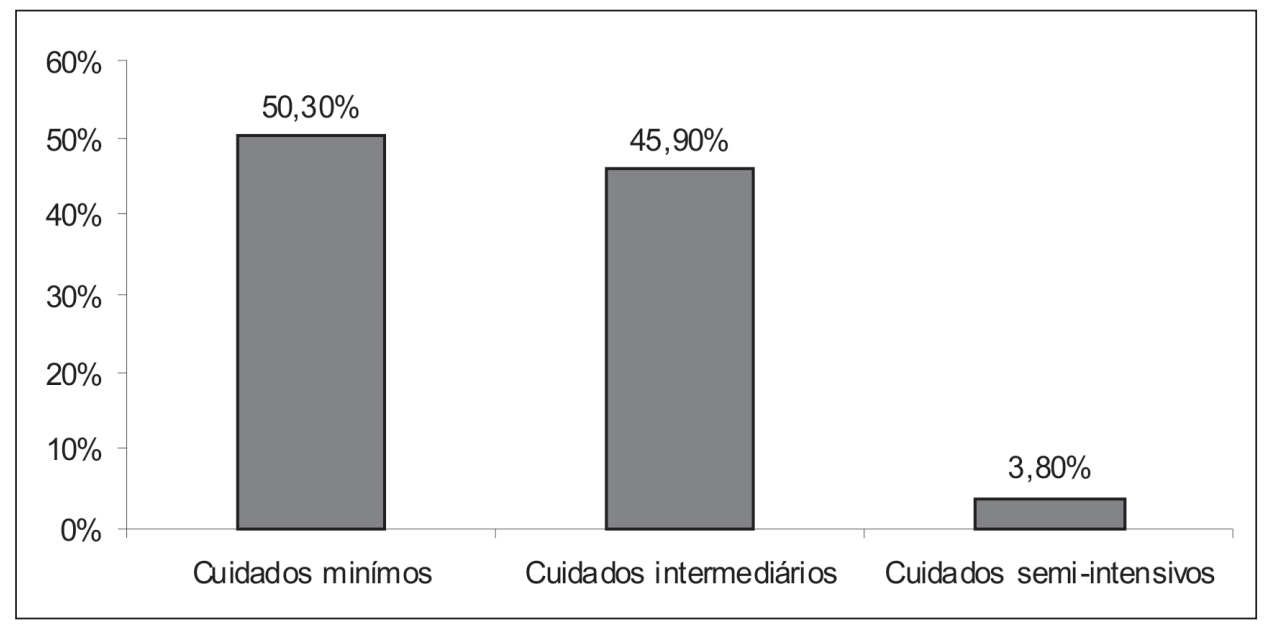

Figura 1 - Sistema de classificação de Perroca. Porto Alegre, RS, 2010.

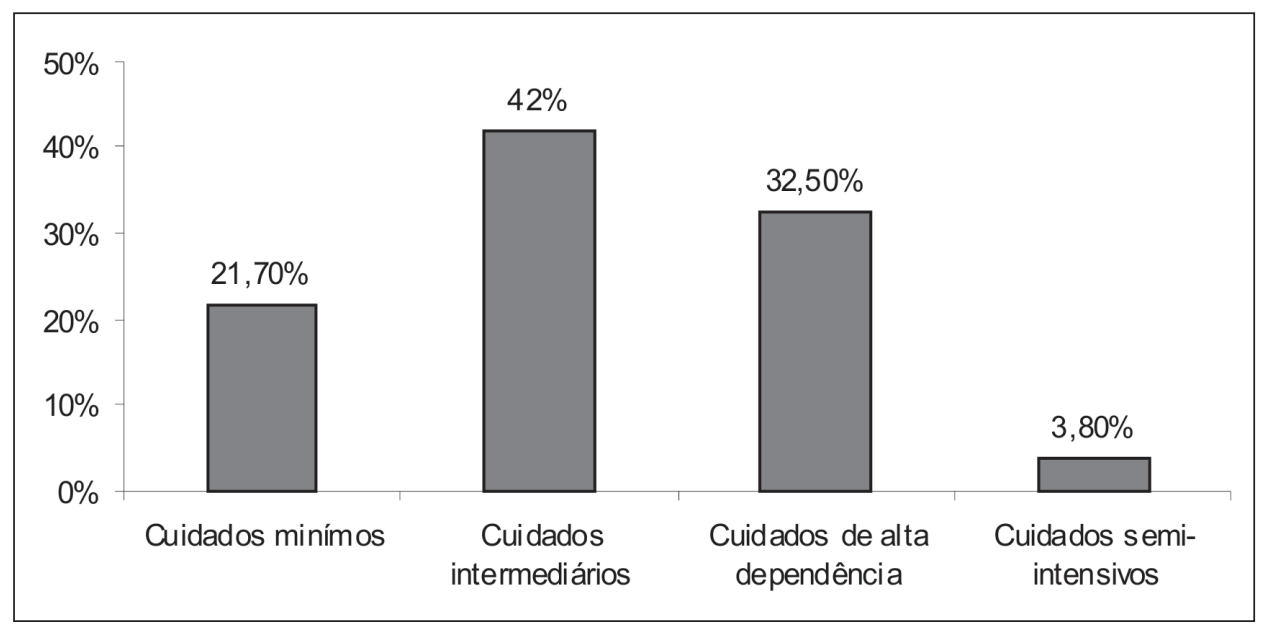

Figura 2 - Instrumento de classificação de Fugulin. Porto Alegre, RS, 2010.

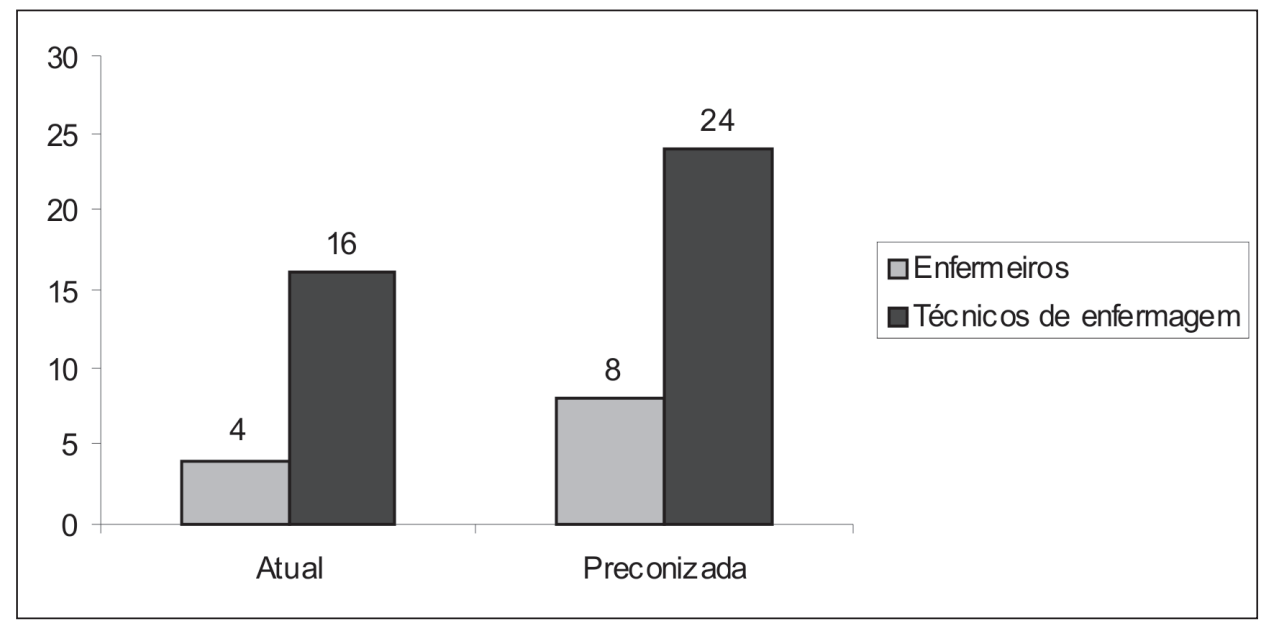

Figura 3 - Dimensionamento da equipe de enfermagem. Porto Alegre, RS, 2010. 
Em relação ao dimensionamento da equipe de enfermagem, consideraram-se os escores obtidos pelo sistema de classificação de Fugulin, em consonância com a resolução COFEN n ${ }^{\circ} .293 / 04^{(5)}$.

Após realizar o cálculo, conforme descrito na metodologia, obteve-se como resultado o número adequado de membros na equipe de enfermagem: seis técnicos de enfermagem e dois enfermeiros por turno. Totalizando 32 membros da equipe de enfermagem para os quatro turnos de trabalho.

\section{DISCUSSÃO}

Este trabalho evidenciou a classificação de pacientes internados em uma unidade de internação traumatológica, de acordo com o grau de dependência da assistência de enfermagem, proposto por Perroca e Fugulin. A amostra constitui-se, majoritariamente, de adultos jovens do sexo masculino, cuja faixa etária é mais atingida por acidentes, sejam eles automobilísticos, de recreação ou esportivo( ${ }^{(6)}$.

No presente estudo, as causas principais de internação foram predominantemente ocasionadas pelos acidentes automobilísticos, seguidos de cirurgias eletivas. Em menor frequência, encontramos as quedas. Em um estudo do Hospital das Clínicas de São Paulo sobre traumatismo da coluna vertebral, as principais causas de internação foram ferimentos por arma de fogo $(36,7 \%)$, acidentes de trânsito $(26,9 \%)$, quedas gerais $(22,4 \%)^{(7)}$. Tal estudo chama a atenção para esses acidentes como uma das causas principais de internação, seguida por ferimentos de arma de fogo e quedas. No Brasil, hospitalizações por acidentes, violência e causas externas ocupam o sexto lugar entre os motivos de internação, representando 8,6\% do total dos gastos públicos com a saúde ${ }^{(8)}$.

Em relação ao diagnóstico médico na internação, foram prevalentes as fraturas ocasionadas por acidentes automobilísticos ou queda. Esses dados expressam a gravidade dos pacientes que são atendidos em um serviço hospitalar de referência em trauma. Estudo ${ }^{(9)}$ realizado com o objetivo de descrever as lesões ortopédicas traumáticas, que incluiu 2.381 pacientes com idade inferior a 20 anos, atendidos no setor de urgência do hospital mostrou uma prevalência de $49,77 \%$ de fraturas, sendo a fratura de rádio a mais prevalente neste estudo.

Por outro lado, um estudo da prevalência de agravos ortopédicos e de suas causas, descreve que as fraturas correspondem a maior parcela dos agravos nos pacientes entre 50 a 70 anos, com predomínio de mulheres, justificando-se pelo envelhecimento, obesidade, sedentarismo e agravos ocupacionais que predispõem a um maior número de encaminhamento à ortopedia. Com relação ao nosso estudo, destacamos o número de fraturas e a faixa etária são próximas, quando comparadas aos estudos mencionados ${ }^{(8)}$.

Acidentes com fraturas ocorrem todos os dias, com qualquer tipo de pessoa, atingindo jovens (acidentes automobilísticos) e idosos (quedas). E muitos destes acidentes levam o paciente a submeter-se a cirurgias reparadoras de alto grau de complexidade ${ }^{(9)}$.

Os crescentes avanços no tratamento destas lesões, devido ao desenvolvimento e treinamento de equipes especializadas no atendimento aos pacientes traumatológicos, nos principais hospitais de referência para o trauma, proporcionam maior expectativa de sobrevida, inclusive nos casos mais graves, além da redução de complicações. No entanto, o prognóstico depende de uma longa fase de reabilitação para a reintegração do indivíduo à sociedade. A melhor conduta, portanto, passa a ser a prevenção.

Com relação ao período de permanência hospitalar, no presente estudo verificou-se que a mediana de tempo foi de 4 dias. Comparando este dado com o outro estudo ${ }^{(10)}$ em que foi aplicado um instrumento de classificação de pacientes em dois períodos distintos, em um setor clínico, evidenciou-se que o período de permanência hospitalar foi de 12,8 dias, sendo que a idade variou de 14 a 88 anos. Observa-se que a amostra do nosso estudo, por ser mais jovem, apresentou um menor tempo de permanência, em virtude da demanda de leitos que é grande no setor pesquisado, e pelo fato da instituição ser referência em trauma e ortopedia na região.

Em relação ao sistema de classificação de Perroca, evidenciou-se que 50,3\% dos pacientes apresentaram complexidade assistencial de cuidados mínimos, 45,9\% de cuidados intermediários, e 3,8\% estão classificados como cuidados semi-intensivos. Um estudo realizado numa unidade cirúrgica de um hospital geral de Porto Alegre observou-se que $70 \%$ dos pacientes necessitavam de cuidados mínimos, $28,7 \%$ de cuidados intermediários e 1,3\% de cuidados semi-intensivos. Também se evidenciou a inexistência de cuidados intensivos de enfermagem para tais pacientes ${ }^{(11)}$.

Em nosso estudo, nota-se um decréscimo no percentual de cuidados mínimos e intermediários, e um aumento no índice de cuidados semi-intensivos, o que leva a inferir que, na unidade traumatológica, 
existem pacientes exigindo maior atenção, no que se refere à prestação de cuidados de enfermagem a pacientes que fazem uso de tração esquelética, fixador externo e restrição ao leito. Da mesma forma, é observada a inexistência de pacientes requerendo cuidados intensivos de enfermagem, fato supostamente esperado, visto que quando ocorre o agravamento do quadro dos pacientes, estes são transferidos para a unidade de terapia intensiva.

Dados de uma pesquisa ${ }^{(10)}$ realizada em unidade clínica hospitalar, onde foi aplicado o instrumento de classificação de Perroca, em dois períodos distintos, com o intuito de conhecer o grau de dependência dos pacientes em relação à equipe de enfermagem, evidenciou que a maioria dos pacientes foi classificada como cuidados mínimos (38,1\%) e cuidados intermediários (25,4\%), sendo preocupante o número de pacientes classificados como cuidados semi-intensivos $(17,5 \%)$ e intensivos (19\%) nas unidades de internação.

Com relação ao instrumento de Classificação de Fugulin, evidenciamos em nosso estudo que 21,7\% dos pacientes, cuidados mínimos, enquanto $42 \%$ dos pacientes estão categorizados como cuidados intermediários, 32,5\% cuidados de alta dependência e 3,8\% cuidados semi-intensivos.

Estudo $^{(4)}$ com objetivo de identificar o perfil assistencial dos pacientes das unidades de internação cirúrgica, durante seis meses, mostrou que o maior número de pacientes foi classificado como cuidados mínimos, seguido por pacientes que necessitavam de cuidados de alta dependência de enfermagem e cuidados intermediários. Já em nosso estudo, podemos comparar os pacientes com cuidados intermediários e de alta-dependência, pois os cuidados mínimos neste estudo foram apenas $21,7 \%$, seguidos de cuidados semi-intensivos, com 3,8\%, ou seja, estes dados afirmam cuidados intermediários e de alta dependência. Salienta-se que a manutenção de pacientes com esse tipo de cuidado demanda maior trabalho e é mais trabalhosa para os profissionais.

A recuperação do paciente e sua menor dependência da enfermagem possibilitam um bom prognóstico, sendo aquele que leva o paciente ao autocuidado, à capacidade do ser humano em atender às suas necessidades básicas após a implementação do plano assistencial ${ }^{(10)}$.

Nas instituições de saúde e, principalmente, nas hospitalares, o serviço de enfermagem desempenha papel fundamental no processo assistencial. Na tentativa de resolver a problemática de cálculo de horas de assistência de enfermagem, tem sido introduzido, no dimensionamento de pessoal, o chamado sistema de classificação de pacientes que permite considerar a gravidade do paciente internado no cálculo de pessoal de enfermagem para o setor.

Neste estudo, o quadro de funcionários, dimensionado de acordo com o preconizado pela legislação específica, é de seis técnicos de enfermagem e dois enfermeiros por turno, conforme a classificação de dependência pelo instrumento de Fugulin, o qual é reconhecido e aprovado pelo COFEN, pela resolução 293/O4 $4^{(5)}$. No entanto, na prática diária, conta-se com apenas quatro técnicos de enfermagem e uma enfermeira por turno, totalizando 20 funcionários. Tal fato evidencia um déficit de aproximadamente 12 funcionários, o que pode gerar sobrecarga de trabalho na equipe atuante, que poderá incorrer em absenteísmo, além do declínio da qualidade do cuidado ofertado.

No setor estudado, por ser específico para internação de pacientes traumatológicos e ainda poli-traumatizados, pacientes com fixadores externos e tração esquelética demandam mais horas de assistência de enfermagem, devido ao seu grau de complexidade. O dimensionamento inadequado dos recursos humanos em enfermagem traz implicações sobre os resultados obtidos pela assistência de enfermagem, em virtude dos aspectos quantitativos e qualitativos de pessoal estarem diretamente ligados à qualidade da assistência prestada ao paciente.

Deve-se salientar que, na prática, o tempo dispensado na assistência de enfermagem para realização de um procedimento, oscila de acordo com as influências da idade, complexidade e colaboração do paciente, além dos recursos materiais disponíveis.

\section{CONSIDERAÇÕES FINAIS}

Na aplicação dos instrumentos de classificação de Fugulin e Perroca, a maior prevalência da classificação do paciente foi a de nível intermediário. Foi avaliado o grau de dependência, observando-se a necessidade do paciente para deambulação, alimentação, higiene, movimentação, eliminações, condições de autocuidado, diagnóstico médico, entre outros. Ainda, ao correlacionar os dois instrumentos obteve-se uma forte correlação $\left(r_{p}=0,88\right)$.

Este estudo verificou o grau de dependência dos pacientes e sua implicação para o dimensionamento de pessoal para assistência de enfermagem no setor de internação traumatológica. É necessário 
que o paciente seja classificado quanto ao grau de dependência, o que contribui para o planejamento das horas de cuidado de enfermagem. Assim, ao se dimensionar os recursos humanos para o cuidado, é possível qualificar o planejamento da assistência de enfermagem, a previsão dos custos e melhor distribuir as atividades entre os membros da equipe de enfermagem.

Cabe mencionar, que há limitação da Resolução COFEN 293/04, devido a mesma não contemplar o nível de cuidados de alta dependência, que consta no sistema de classificação de Fugulin.

\section{REFERÊNCIAS}

1 Fonseca JP, Echer IC. Grau de dependência de pacientes em relação à assistência de enfermagem em uma unidade de internação clínica. Rev Gaucha Enferm. 2003; 24(3): 346-54.

2 Perroca MG, Gaidzinski RR. Sistema de classificação de pacientes: construção e validação de um instrumento. Rev Esc Enferm USP. 1998; 32(2): 153-68.

3 Perroca MG, Gaidzinski RR. Análise da validade de constructo do instrumento de classificação de pacientes proposto por Perroca. Rev Latino-Am Enferm. 2004; 12(1): 83-91.

4 Fugulin FMT, Gaidzinski RR, KurcganT P. Sistema de classificação de pacientes: identificação do perfil assistencial dos pacientes das unidades de internação do HU-USP. Rev Latino-Am Enferm. 2005; 13(1):72-8.

5 Conselho Federal de Enfermagem. Resolução $n^{\circ}$ 293/04: estabelece parâmetros para o dimensionamento do quadro de profissionais de enfermagem nas instituições de saúde. [Internet]. Brasília(DF):
COFEN; 2004 [citado 2010 mai 03]; Disponível em: http://site.portalcofen.gov.br/sites/default/files/ RESOLUCAO2932004.PDF

6 Dellatorre MCC, Cazzo E, Silva VA, Yanagitani, VK, Carvalho FF. Distúrbios ortopédicos e traumatologia: análise de 5.330 casos em unidade de urgência e emergência. Rev Bras Med. 2001; 8 (1): 73-77.

7 Barros FTEP, Taricco MA, Oliveira RP, Greve JMA, Santos LCR, Napoli MMM. Estudo epidemiológico dos pacientes com traumatismo da coluna vertebral e déficit neurológico, internados no Instituto de Ortopedia e Traumatologia do Hospital das Clínicas da Faculdade de Medicina da USP. Rev Hosp Clin Méd USP. 1999: 45(3):123-6.

8 Hurba ABM, Deus RB, Barnabé AS, Oliveira RS, Ferraz RRN. Prevalência de agravos ortopédicos e de suas causas em uma população da região de São Paulo. Conscientiae Saúde. 2009; 8(2): 251-7.

9 Carvalho Júnior LH, Cunha FM, Ferreira FS, Morato AEP, Rocha LHA, Medeiros RF. Lesões ortopédicas traumáticas em crianças e adolescentes. Rev Bras Ortop [Internet]. 2000 mar [citado 2009 jul 10]; 35 (3):80-87. Disponível em: http://www.rbo.org. $\mathrm{br} /$ materia.asp? $\mathrm{mt}=579 \&$ id Idioma $=1$

10 Carmona PML, Évora MDY. Grau de dependência do paciente em relação à enfermagem: análise de prontuários. Rer Latino-Am Enfermagem. 2003;11 (4): 468-73.

11 Lopes ACS. Classificação do grau de dependência de pacientes em uma unidade de internação cirúrgica de um hospital geral universitário de Porto Alegre [trabalho de conclusão de curso]. Porto Alegre: Curso de Graduação em Enfermagem, Escola de Enfermagem Universidade Federal do Rio Grande do Sul; 2000.

\section{Endereço do autor / Dirección del autor / Author's address:}

Emiliane Nogueira de Souza

Universidade Federal de Ciências da Saúde de Porto Alegre

Rua Sarmento Leite, 245/717, Anexo II, Farroupilha 90050-170, Porto Alegre, RS

E-mail:emilianes@ufcspa.edu.br 